\title{
ESTUDIO MORFOLÓgICO DEL POLEN DE BORDASIA BICORNIS (MALVACEAE)
}

\author{
GRACIELA ANA CUADRADO
}

\begin{abstract}
Summary: Cuadrado, G.A. 2003. Pollen morphological study in Bordasia bicornis (Malvaceae). Bonplandia 12(1-4): 137-140. ISSN: 0524-0476.

Pollen grains of Bordasia bicornis Krapov. were studied and described with light and scanning electron microscopy. They showed palynological affinity with species of genus Sida L, therefore they were compared with 39 species of Argentina, Brasil, Colombia. Mexico, South Africa, India, Thailand, Philippines, Texas and Florida U.S.A. Bibliographic and personal data were used. Affinities in the structure of the exine and height of the spines as well as differences in apertures number and pores without thicker ring were found.
\end{abstract}

Key words: Pollen, Sida, South America.

Resumen: Cuadrado, G.A. 2003. Estudio morfológico del polen de Bordasia bicornis (Malvaceae). Bonplandia 12(1-4): 137-140. ISSN: 0524-0476.

Fueron estudiados y descriptos los granos de polen de Bordasia bicornis Krapov. con microcopio óptico y microscopio electrónico de barrido. Éstos presentaron afinidad polínica con especies del género Sida L, por lo que se compararon con 39 especies de Argentina, Brasil, Colombia, México, Sud África, India, Tailandia, Filipinas Texas y Florida, EE.UU. del citado género, usando información bibliográfica y de la propia autora. Se hallaron afinidades en la estructura de la exina y altura de las espinas y diferencias como el número de aperturas y poros sin engrosamiento.

Palabras claves: Polen, Sida, América de Sur.

\section{Introducción}

Se estudiaron los granos de polen de Bordasia bicornis, nuevo género y especie creados por Krapovickas (2003). El autor expresa la afinidad del nuevo taxón con el género Sida, refiriéndose a la semilla solitaria y péndula y al cáliz sin calículo y lo diferencia de él por caracteres de los mericarpos, hojas y forma del cáliz.

Se han comparado los granos de polen de Bordasia bicornis con los de 39 especies del género Sida, 21 de la Argentina: Sida anómala A.St.-Hil., $S$. argentina K.Schum., $S$. calchaquiensis Rodrigo, S. confusa Hassl., S. cordifolia L., S. dictyocarpa Griseb., S. dubia A.St.-Hil. \& Naudin, S. glutinosa Cav., $S$.

Cátedra de Palinología- Facultad de Cs. Exactas y Naturales y Agrimensura- UNNE. Centro de Ecología Aplicada (CECOAL)-CONICET.Ruta 5 km 2,5-C.C.291-(3400) Corrientes- Argentina. Tel/Fax 54-(03783)454417. E-mail: hcaplan@amet.com.ar 
nemorensis Mart, ex Colla, $S$. paradoxa Rodrigo, $S$. potentilloides A.St.-Hil., $S$. regnellii R.E.Fr., $S$. rhombifolia L., $S$. rodrigoì Monteiro, $S$. santaremensis Monteiro, S. spinosa L., $S$. tuberculata R.E.Fr., $S$. variegata (Griseb.) Krapov., S. vespertina Ekman, S. viarum A.St.-Hil., S. urens L. (Cuadrado, Tesis en preparación), 7 de Texas (EE.UU.): S. ciliaris L., S. füicaulis Torrey \& A.Gray., $S$. filipes A. Gray, $S$. lindheimeri Engelm. \& A.Gray, S. neomexicana A.Gray, S. tragiaefolia A.Gray, S. longipes A.Gray (Hashmi, 1970), 3 especies de México: $S$. acuta Burm., S. linifolia Juss. y S. tragiaefolia A.Gray (Sánchez Martínez, 1982) y 8 de Brasil, India, Sud África, Florida (EE.UU.), Tailandia y Filipinas respectivamente: $S$. acuta Burm., S. angustissima A.St.-Hil., $S$. chrysantha Ulbr., $S$. ciliaris, $S$. cordifolia, $S$. elliotii Torrey \& A.Gray, $S$. glutinosa y $S$. veronicaefolia Lam. (Christensen ,1986).

\section{Bordasia bicornis Krapov.}

Fig. 1

Granos esferoidales muy grandes, de 102 (107) $112 \mu \mathrm{m}$ de diámetro. Poros en número de 7-8, de $6 \mu \mathrm{m}$ de diámetro en disposición helicoidal y con engrosamiento anular de 4 $\mu \mathrm{m}$. Distancia interporal aproximadamente 25 $\mu \mathrm{m}$. Exina de $3 \mu \mathrm{m}$ de espesor en el área subespinal y $2 \mu \mathrm{m}$ en la zona interespinal. Sexina de $2 \mu \mathrm{m}$ bajo las espinas y $1 \mu \mathrm{m}$ entre las espinas. El mayor espesor de la sexina, bajo las espinas está dada por la mayor altura de las báculas, que determinan a este nivel los clásicos "mamelones del polen de Malvaceae. La nexina, se mantiene constante, aumentado su espesor sólo a nivel de los poros donde forma el engrosamiento que los rodea. El tectum es muy delgado de aproximadamente $0,5 \mu \mathrm{m}$. Espinas de $4 \mu \mathrm{m}$ de altura, densamente dispuestas, con una distancia entre ellas de $5 \mu \mathrm{m}$, alternando con elementos positivos menores de $1 \mu \mathrm{m}$.

MEB: Entre las espinas se observan perforaciones circulares (fóveas) y espínulas.

Material estudiado: Paraguay. Alto Paraguay, Línea 1, km 1336. Pérez de Mola 8466 (CTES), PAL-CTES 6709.

\section{Comparación con las especies citadas del género Sida}

Se hallaron caracteres comunes en cuanto a la estructura de la exina, el tamaño y forma de las espinas y la distancia entre las mismas. Caracteres de Bordasia bicornis y de especies comparadas del género Sida están detallados en el siguiente cuadro:

Tamaño de los granos: muy grandes $(102-112 \mu \mathrm{m})$ Tamaño de los granos: grandes y muy grandes $(50-107,3 \mu \mathrm{m})$ Número de aperturas: 7-8

En cuanto a las especies de Sida de Argentina, los granos son grandes $(50-88 \mu \mathrm{m})$. El número de poros es de 12-30; la distancia entre ellos 10-18 $\mu \mathrm{m}$. Sólo dos especies: $S$. potentilloides y $S$. nemorensis tienen poros con engrosamiento anular, pero de $2 \mu \mathrm{m}$. Las demás especies no presentan tal engrosamiento (Cuadrado, inéd.).

Los granos de polen de las especies de Texas son oblato esferoidales, grandes $(69,35$ x $77 \mu \mathrm{m})$, trizonocolporados y esferoidales grandes $(61,5-62,61 \times 81,34-83,88 \mu \mathrm{m}) \operatorname{con} 8$ 

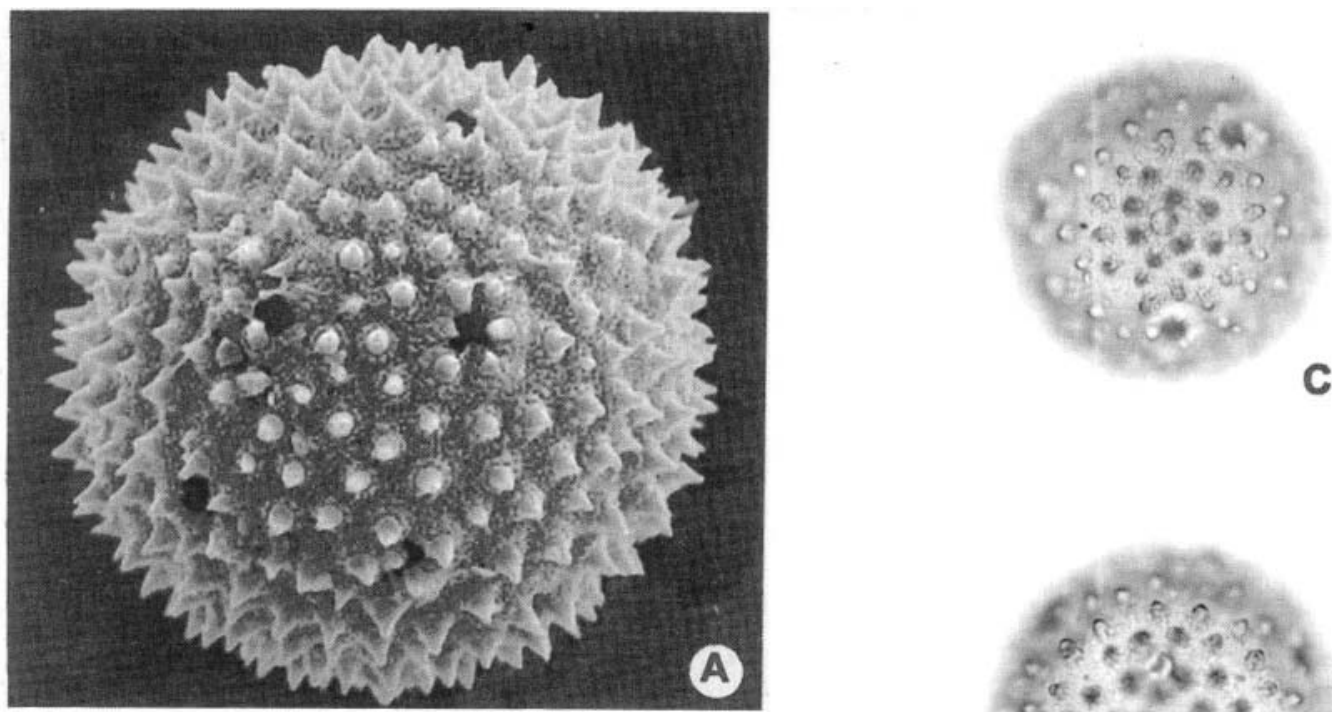

C
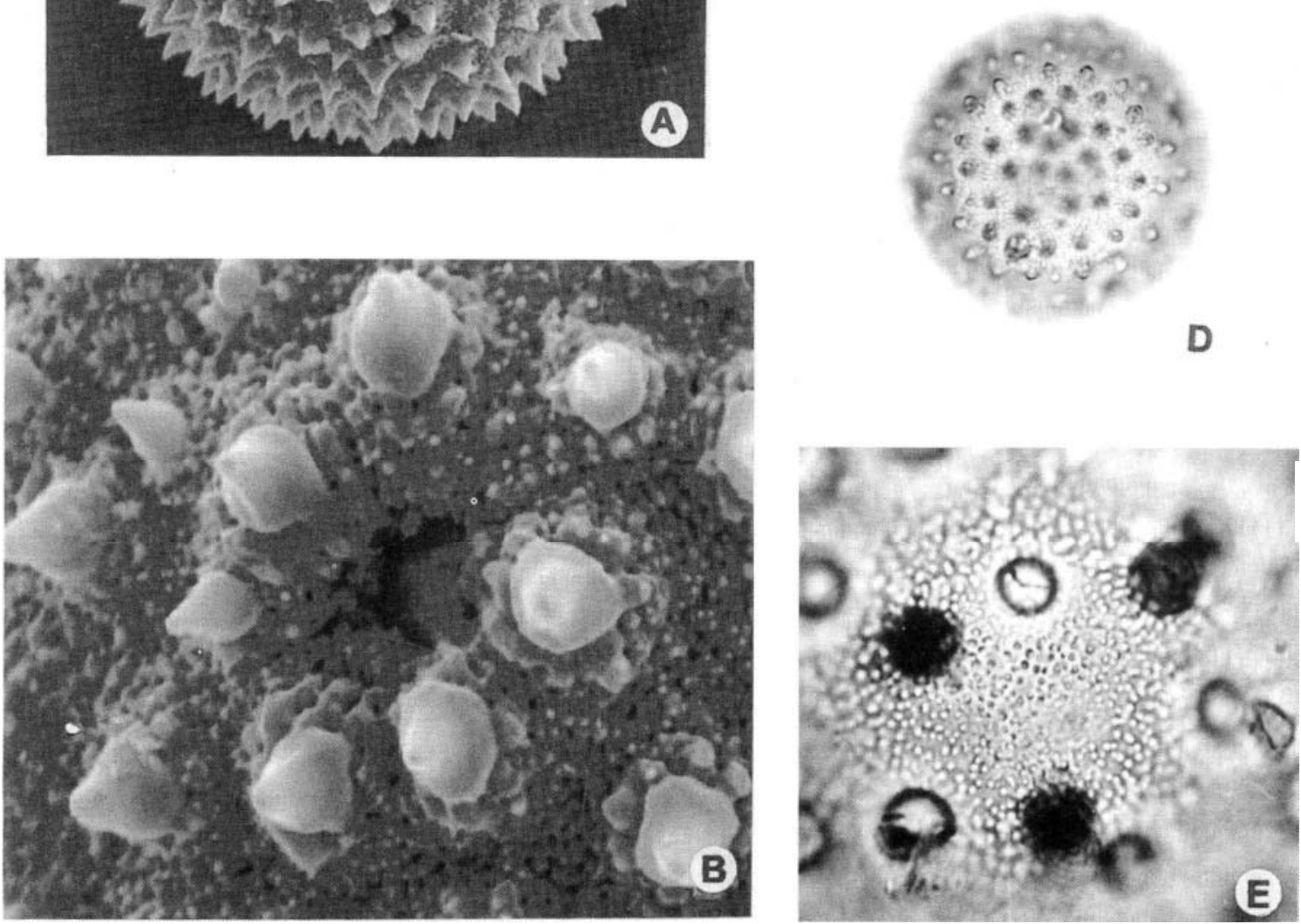

Fig. 1: Bordasia bicornis. MEB: A: vista general mostrando cinco aperturas (poros). B: detalle de escultura y apertura. MO: C: vista general mostrando tres aperturas. D: vista general mostrando cuatro aperturas. E: detalle de escultura y aperturas. La escala equivale a $32 \mu \mathrm{m}$ en $\mathrm{A}$, a $14 \mu \mathrm{m}$ en B, a 62 u.m en C y D, y a 30 u.m en E. 
-35 pantoaperturas, que pueden ser poros o colporos según las especies. En una sola, $S$. tragiaefolia, el tamaño de los granos es levemente mayor que en las demás (101 a $103 \mu \mathrm{m})$ y en este carácter se superpone con las medidas de Bordasia bicornis, pero al presentar un alto número de poros (25 a 35 ), se diferencia de ella. La distancia interporal es de 12 a 19,8 pin, con excepción de $S$. neomexicana donde es de $27,3 \mu \mathrm{m}$, pero el tamaño de los granos es grande $(81,4 \times 83,8 \mu \mathrm{m})$ y las aperturas son colporadas, a diferencia de la especie estudiada que tiene granos muy grandes y son pantoporados (Hashmi, 1970).

Ninguna de las especies de Sida de Texas estudiadas por Hashmi presenta engrasamiento en los ora, en caso de los colporados, ni en los poros en el caso de los porados.

La descripción de Christensen (1986) del polen de 8 especies de Sida fue realizada en conjunto. Estos granos son grandes $(62 \times 60$ $76 \times 70)$. Las aperturas son 6-90 colporados y porados. La autora, si bien no discrimina los caracteres entre especies, aclara que los granos de polen oligoaperturados son los que tienen aperturas compuestas y los multiaperturados los que tienen aperturas simples. Por lo que se puede inferir que los granos de polen con menos de 8 aperturas, que es el número que coincide con Bordasia bicornis, son colporados, y en esta especies son porados. O sea, que aunque coincidan en número de aperturas, difieren en el tipo de las mismas. También se diferencian por el tamaño de los granos.

En cuanto a las tres especies mexicanas de Sida descriptas por Sánchez Martínez (1982), todas tienen afinidad entre sí y con la especie estudiada en cuanto a la forma de los granos y el tipo de apertura, el autor no menciona el número de aperturas en ningún caso. El tamafío de los granos sólo diferencia a $S$. acuta, ya que son grandes de 57,6 a $66,7 \mu \mathrm{m}$, las otras dos especies $S$. linifolia y $S$. tragiaefolia son grandes y muy grandes de 94,3 a 107,3 $\mu \mathrm{m}$, rango en el que está comprendida Bordasia bicornis.

Teniendo en cuenta las comparaciones realizadas puede afirmarse que Bordasia bicornis es muy próxima a las especies de Sida, por algunos de sus caracteres palbiológicos: forma, tipo y altura de las espinas y estructura de la exina. Otros caracteres como tamaño de los granos, número de aperturas, distancia entre las mismas y ausencia de engrasamiento anular en los poros u ora, en la mayoría de las especies comparadas, diferencian a Bordasia bicornis del género Sida.

\section{Bibliografía}

CUADRADO, G.A. Palinología vinculada a la irrupción del picudo del algodonero (Anthonomus granáis B.) en el nordeste argentino. (Tesis en preparación).

CHRISTENSEN, P.B. 1986. Pollen morphological studies in the Malvaceae. Grana 25(2): 95-117.

HASHMI, S.H. 1970. The Palynology of the Malvaceae of Texas. Submitted to the Graduate College of Texas A \& M University in Partial fulfillment of the requirement for the degree of Doctor of Philosophy.

KRAPOVICKAS, A. 2003. Bordasia Krapov., nuevo género de Malváceas. Bonplandia 12(1-4): 133135.

SÁNCHEZ MARTÍNEZ, F. 1982. Morfología polínica de algunas Malváceas mexicanas. Instituto de Antropología e Historia. Centro del Sureste. Colección científica 127. México. 